\title{
Mechanism of Quantum Consciousness that Synchronizes Quantum Mechanics with Relativity-Perspective of a New Model of Consciousness
}

\author{
Siva Prasad Kodukula (1) \\ Independent Researcher, Visakhapatnam, India \\ Email: sivkod@gmail.com
}

How to cite this paper: Kodukula, S.P. (2021) Mechanism of Quantum Consciousness that Synchronizes Quantum Mechanics with Relativity-Perspective of a New Model of Consciousness. Journal of Modern Physics, 12, 1633-1655.

https://doi.org/10.4236/jmp.2021.1212097

Received: September 11, 2021

Accepted: October 17, 2021

Published: October 20, 2021

Copyright (อ 2021 by author(s) and Scientific Research Publishing Inc. This work is licensed under the Creative Commons Attribution International License (CC BY 4.0).

http://creativecommons.org/licenses/by/4.0/ (c) (i) Open Access

\begin{abstract}
Synchronization of quantum mechanics with relativity has been considered differently from the present quantum gravity models. It is originated from the roots of philosophy of physics and the basic concepts of relativity \& quantum mechanics. It emphasizes the fact that two conscious observers are necessary to experience one conscious moment. Various concepts of consciousness have been discussed and emphasized the necessity for the introduction of a new model of quantum consciousness. A quantum coordinate system has been introduced to explain the present understanding of the phenomena "observation" and "reality". It has been elaborated that the observation defined by physics is confined to Lorentz space time coordinate system, Minkowski coordinate system and general relativity. But phenomena of observation cannot be completed without considering one more hidden transformation explaining quantum coordinate system which transforms the quantum states into relativistic coordinate system as an interaction between two conscious observers explained by an interactive mechanism of quantum states. A flow chart has been illustrated by a mechanism giving rise to conscious moment and proposed a new model of consciousness. It emphasizes on the fact that "reality" is different from "observation" defined by physics. It affects the relativistic factor of special relativity and suggests a modification for it. If this modified relativistic factor is proved experimentally, the results establish consciousness's mechanism and a remarkable breakthrough in physics of consciousness studies.
\end{abstract}

\section{Keywords}

Quantum Mechanics, Relativity, Double Relativity Effect, Film Theory of 


\section{Introduction}

Fundamentally quantum mechanics is not synchronizing with General relativity because, at quantum level i.e. beyond a limit, General relativity equations cannot explain space time. Quantum mechanics describes discreteness of space time and General Relativity interprets continuous and smooth space time. These two are not in synchronization.

\subsection{Present Quantum Mechanics}

Wave theory of light has been introduced in 17th century. Double slit experiment proposed in 1803 by Thomas young played an important role in establishing wave theory. In 1900 Planck proposed quantum theory. In 1905 Einstein explained photo electric effect by Planck's quantum theory. Modern quantum mechanics originated after the introduction of de Broglie's equation explaining the wave nature of particle in the years 1923 to 1925 .

Later, matrix mechanics was introduced. Schrödinger wave function was introduced in 1926. By 1930 quantum mechanics had been further unified and formalized by David Hilbert, Paul Dirac and Neumann with greater emphasis on measurement, the statistical nature of our knowledge of reality and definition of "observer". Even today, Measurement problem, observables and "observer" plays an important role in the development of quantum theory [1] [2] [3].

Observer and observation has a deeper meaning involving the concept of consciousness [2] [4]. In this paper it is explained that without observers there is no meaning for the word "Reality" described by physics. Of course the reality is linked with Relativity also.

\subsection{Relativity}

Galilean or Newtonian transformations are equations that relate space and time coordinates of two systems moving with constant velocity relative to each other. It is failed to interpret light velocity which was described by Maxwell's equations. They are not invariant in Galilean transformations.

\subsection{Lorentz Transformation}

According to Lorentz transformation, the observers moving at different velocities may measure different distances such that the velocity of light is the same in all inertial reference frames. This invariance of light velocity has been considered as a postulate of special theory of light.

\subsection{Special Relativity}

In the year 1905 special theory of relativity has been published. It has elaborated 
the conventional notion of an absolute universal time with the notion of a time that is relative to reference frame and its position in space. Rather than an invariant time interval between two events, there is an invariant space time combined with other laws of physics and proposed the mass energy equivalence principle. Special relativity interprets a flat four-dimensional Minkowski space time. It appears to be very similar to the standard Three-dimensional Euclidian space, but there is a difference with respect to time. It has reduced the spatial dimensions in to two, so that we can represent the physics in a three dimensional space. In Newtonian mechanics, quantities that have magnitude and direction are mathematically described as three dimensional vectors in Euclidean space, and in general they are represented by time. In special relativity, this notion is extended by adding the appropriate time like quantity to a space like vector quantity, and we have four dimensional vectors, or "four vectors" in Minkowski space time. The components of vectors are written using tensor notation. In Newtonian gravity, the source is mass. In special relativity, mass turns out to be part of a more general quantity called the energy-momentum tensor which includes both energy and momentum as well as stress pressure and sheer. Using the equivalence principle, this tensor is readily generalized to curved space time.

\subsection{General Theory of Relativity}

Thus in 1915 General relativity proposed. According to this theory, there is no gravitational force deflecting objects from their natural, straight paths. Instead, gravity tends to changes in the properties of space and time, which in turn changes the straightest-possible paths that objects will naturally follow. The curvature is, in turn, caused by the energy-momentum of matter. As it is constructed using tensors, general relativity exhibits general covariance. It thus satisfies a more stringent general principle of relativity, says that the laws of physics are the same for all observers. In other words, as expressed in the equivalence principle, space-time is Minkowiskian and the laws of physics exhibit Lorentz invariance.

If we consider General relativity as most fundamental and explainable by geometry, quantum theory the basis of understanding matter from elementary particles is unexplainable by space time geometry at quantum level. However, how to reconcile quantum theory with general relativity is still an open question. In order to explain the importance of the problem, few authors conducted a survey and published the results [5].

\subsection{Relativistic Quantum Mechanics}

Relativistic quantum mechanics is application of special relativity for quantum particles. It is not a theory to reconcile quantum mechanics with general relativity. Dirac equation is resultant of this concept. Based on this concept, super fluid theories have been proposed [6].

According to General Relativity the conventional gravitational wave is: 
1) A small fluctuation of curved space time;

2) It has been separated from its source and propagates independently.

These cannot be completely justified in a theory with exact Lorentz symmetry. They are not perfectly described by relativistic theory.

In this paper the conceptual and physical interpretation of quantum coordinates, in to Lorentz or Minkowski's space time have been explained and the space time incorporated in general relativity. Thus mathematical interpretation of space time curvature is possible by the concept of physical transformation of quantum states represented by quantum coordinates to space time coordinate system of reality. This paper has no relation to super fluid relativity. But it is based on a previous calculation of space time diameters for all fundamental forces [7]. All these space time diameters are interpreted as points with zero space in quantum coordinates in order to obey the property of signal required for transformation.

\subsection{Synchronization of QM with GR}

General relativity equations describe space time curvature. When it is applied to black holes, the physical quantities such as space time divergence at the centre of black holes, when it goes closer to the centre, less than Planck length distance, there is a breakdown of General Relativity (GR) equations. There must be a new theory which goes beyond GR is required and quantum influence plays dominant role. Thus quantum gravity theories originated [8].

\subsection{Loop Quantum Gravity (LQG)}

The main result of loop quantum gravity is the derivation of a granular structure of space at the Planck length. The quantum state of space time is described in the form of spin networks. Much of the work in "loop quantum gravity" or "quantum geometry" area has been based on Dirac quantization of the constraints, though there have been recent advances in the use of covariant "spin foam" methods [8].

All the above models are based on space time geometries, renormalization [8] [9], space time coordinates defined by Newtonian, Galilean, Lorentz etc. But nowhere it is not mentioned that how does this space time forms. The definition of observer which is a part of these coordinate systems is really in the same system? How are we transforming these systems in to one another without knowing the presence of observer whether it is inside or outside of the system? This probe will lead us to formulate a new approach for synchronizing quantum mechanics with general relativity. "Physics of consciousness" emphasizing on these aspects. Involvement of "consciousness" plays a vital role in the synchronisation of quantum mechanics with general relativity. Present studies on consciousness explains as follows.

\subsection{Consciousness}

Consciousness is an interdisciplinary concept with quantum mechanics, relativ- 
ity, space time structure, and biology. Lots of theories are there to define consciousness. But in this paper, based on earlier work, physics of consciousness especially on "measurement problem", "observer effect", and "wave function collapse" have been considered for further probe in to fundamental physics. OrchOR [10] is one of the proposals on this subject. According to that, consciousness is associated to Orchestral Reduction process and is related to gravity. Threshold time to form gravitational space time at basic level, has been calculated [7]. This is the first step to synchronise quantum mechanics with relativity since the quantum particle of our four dimensional space time i.e. gravity is obeying both quantum mechanics and general relativity principle. This has leaded me to propose new hypotheses of consciousness [11]. Some of the experiments in biology [12] are promising for the proof of this direction of thought. Of course, the author expressed it as a proof of Orch-OR theory. The same can be applied further to new hypothesis of consciousness [11] also and the model elaborated in the present paper regarding the physics part of that biological model of consciousness.

\subsection{New Hypothesis of Consciousness}

Analysis on some of the concepts involved in Orch-OR proposal such as threshold time, quantum de-coherence, entanglement, system and the environment with which system interacts etc and the process connecting all these phenomena raised so many alternative solutions for connecting concepts and alternative proposals for integration of them. Thus a new hypothesis explaining the model for "consciousness and information processing" has been proposed [11]. Now, in the present paper, a new model of consciousness has been proposed to interpret "consciousness" in terms of physics. It is completely different from the existing models but similar to Orch-OR proposal.

All the above theories tried to explain this synchronization on the basis of General relativity. But special relativity plays a vital role in this synchronization. Special relativity is a simple explanation to transformations from Newtonian, Galilean to Lorentz. Finally Lorentzian manifold has been transformed in to minkowiski space time manifold which is the basis for general relativity. Quantum mechanics is a parallel development and was not based on these transformations. So general relativity is not in synchronization with quantum mechanics. Loop quantum gravity theories tried to synchronize general relativity with quantum mechanics directly from general relativity. Even though they considered the synchronization through Lorentzian manifold, it has not considered the fact of observation and its transformation which is basically connected to consciousness. If we consider the involvement of consciousness in all these transformations, Newtonian, Galilean and Lorentz transformations, we can understand that one transformation is missing. This transformation has been interpreted by quantum coordinate system. Mechanism of consciousness plays an important role in this synchronization. Of course, without double relativity ef- 
fect [13] [14] [15] it is not at all possible to get an overall view of quantum coordinates.

Explaining all these transformations along with the mechanism of consciousness again one by one, we can find a new way of interpretation for this synchronization. This interpretation will definitely transform the classical or relativistic concepts and quantities in to quantum mechanical system.

\section{Theory \& Discussion}

Established theoretical background of physics explains the space time notions and the representation of its coordinate systems from Newtonian mechanics to General Relativity. Their transformations also have been well established. Now we are introducing a new coordinate system termed as "Quantum Coordinates" in this sequential order. Before introduction of this coordinates, the sequential order is Newtonian, Galilean, Lorentz, Minkowski and General Relativity. Sequential order means without the previous one, the next one is not possible. After introduction of Quantum coordinates, the sequential order will be changed to Newtonian, Galilean, Lorentz, Quantum and Minkowski or General Relativity. Let us see the interpretation of quantum coordinates in transformations.

\subsection{Quantum Coordinate System}

A quantum coordinate system is also like Minkowski or general relativity coordinate system. In that, space will be $\mathrm{X}$ coordinate and time will be $\mathrm{Y}$ coordinate. Figure 1 shows the quantum coordinate system in terms of relativistic coordinates and its interpretation with respect to relativistic coordinates.

But the difference is in quantum coordinates; all points will have zero space. Time only will exist. But in the interpretation of coordinate system, space axis will be existed. Means, even though, space exists, it is considered as zero only. In conventional coordinate system a point will have both time and space where as time only will exist in quantum coordinate system. But the space will be shown as information. All the points will have different time and different information but with space zero. So instead of space axis there exists information axis when comparing with conventional relativistic coordinate system. As per space time equivalence principle, space converts in to time [16]. At the same time, it (time) is nothing but information [11]. So finally, a point will contain time only and as per its position in the coordinate system, it will have time and information. Its space is zero. This time and information describes that point. Since space is zero, it can not contain any object like mass. In the transformation of this coordinate system to space time coordinates, it will be in synchronization with general relativity with space time coordinates and the general relativity equations will be applied. Figure 2 illustrates the observers view of an object through quantum phenomena represented by quantum coordinates.

\subsection{Transformation of Quantum Coordinate System}

For any Lorentz coordinate system, if time changes and space remains constant 


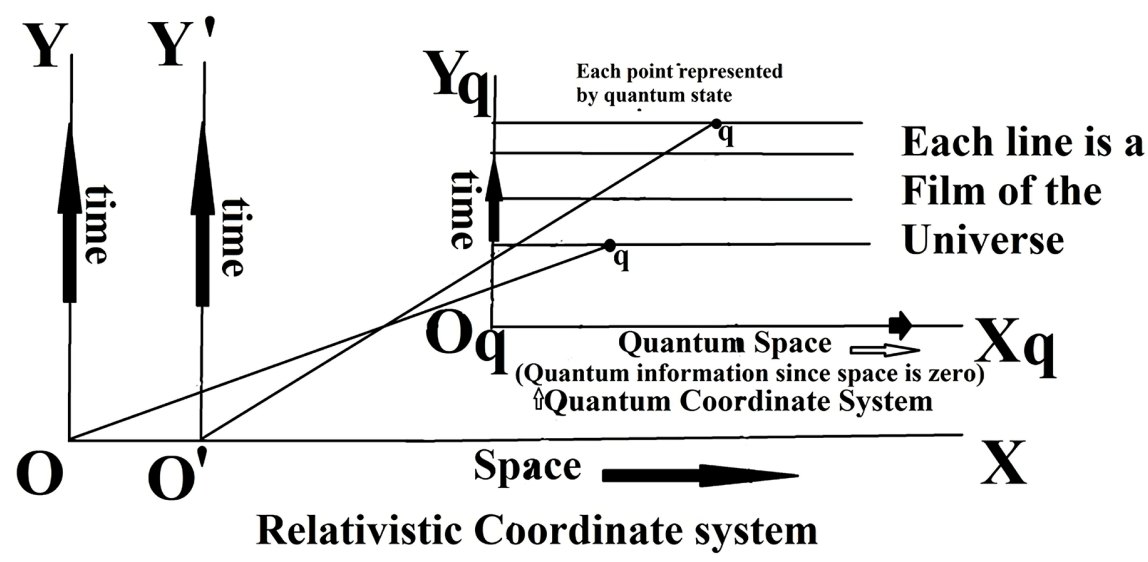

Figure 1. Quantum coordinate system and its interpretation with respect to relativistic coordinate system. Each point represents quantum state and each line represents film of the universe so that the universal film is a superposition of quantum states.

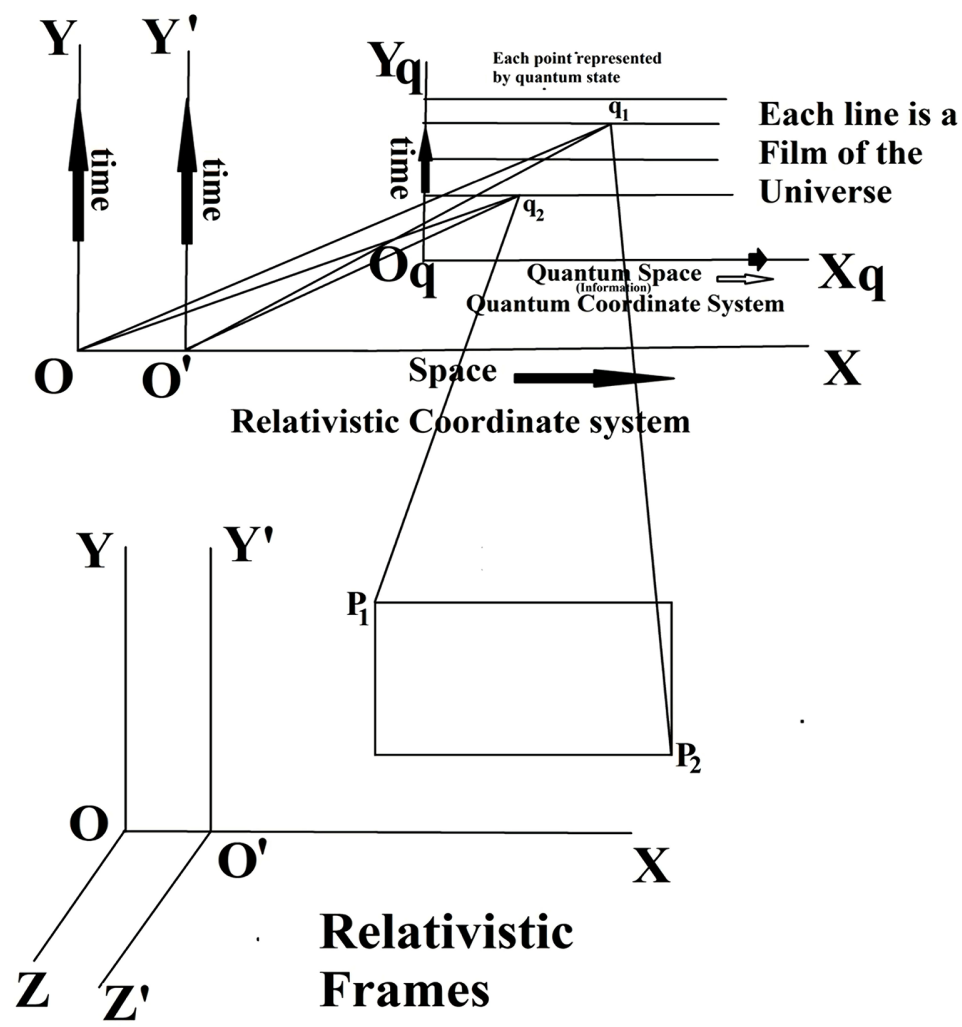

Figure 2. Observer's view of an object through quantum phenomena represented by quantum coordinates. Observer is confined to conventional relativistic frames of reference.

or space changes and time remains constant for a particular frame of reference with velocity, means that frame (or particle) is with a constant velocity relative to all inertial frames of reference. For example, if we observe a particle with velocity " $v$ ", it will cover a distance within a duration of time and at the same time if the observing system moves with velocity, then the relative velocity will be different. But if it is not changed, then change in velocity is zero. If change in veloc- 
ity is zero, change in either of space or time will be zero. Let us suppose the case that time exists and the space (covered distance) is zero as explained above, then its velocity is constant for all inertial frames of reference. Such particles will not exist in this Lorenz coordinate system. Only velocity exists. Any particle in this world occupies space and can be considered as "combination of particles". So "constant velocity relative to each other" has no meaning for that "combination of particles or mass" and such particles will not exist in this conventional space time coordinate system (or Lorentz coordinate system).

Long back author explained the same phenomena by "double relativity effect" and NC particles [13] [14]. Now in this, it is applied for profound understanding of the relation between quantum states and their interpretation in transformation of relativistic frames.

\subsection{Double Relativity Effect}

Double Relativity effect is an effect attributable to absolute velocities. While observing a particle with constant velocity relative to all inertial frames of reference (Light particle or a travelling photon is an example for it). In this, special relativity will be applied twice in a single observation at the same time [13] [14] [15].

Here, the velocity considered in the first stage is absolute velocity and will be effected by a phenomena called "double relativity effect". Thus the absolute velocity will be changed to observed velocity. Double relativity effect elaborates that the Absolute velocity and observed velocity is related by equation $v_{o}=v_{a} \gamma$ where $\gamma=\frac{1}{\sqrt{1-\left(\frac{v_{a}}{c}\right)^{2}}}$.

Any velocity in this universe must pass through infinitely series of points in relativistic space time coordinates. These points are imaginary only. But when object changes through these points in the course of its velocity, the double relativity effect will be applied to that object at that point in coordinate system. So observed velocity is $v_{o}=v_{a} \gamma$.

\subsubsection{Role of Observer}

The paper [17] explains the role of observer and consciousness on special relativity. In this paper, it is elaborated how does an observer considers the object by the application of absolute velocities concept and how films of the universe changes with time does. Film theory' of the universe has been applied and much more elaborated in the paper [11]

\subsubsection{Film of the Universe}

A film of the universe is an inertial frame of reference containing same time interval all over the film as per the film theory of the universe [11] [13].

Further when it is applied to Universe, It is postulated that whole universe is existed with points containing absolute velocity [16]. This velocity is constant for all inertial frames of reference and an absolute velocity " $V$ " exist at distance $d$ 
from an observer and related by equation $v=H d$. At the same time it will have a velocity $v d=K$. The resultant is the observed velocity. Here " $H$ " is Hubble's constant and " $K$ " is Siva's constant.

The same concept is applicable to space time and showed that all the fundamental forces are made up of space times but relatively with different space time densities [7]. Interaction between these fundamental fields with separate space times giving raise to new particles. Space time conversion in to matter is also explained [16]. Here it is connected to Quantum physics and emphasizes the fact that "signal in a space time is nothing but the least diameter of space time in that field". Space time diameters for all fundamental forces have been calculated in the previous works [7].

Now let us see how these concepts can be interpreted by quantum mechanics.

\subsection{Application to Quantum Mechanics}

The physical meaning of quantum state has been explained as per film theory of the universe. [11] [17]. As per film theory of the universe, a film is an inertial frame of reference in which every point shows the same clock. But as per absolute velocities and double relativity effect, the points in space time relativistic coordinates will have different Inertial Frames of references (IFRs) and every point is a representation of universal film with a specific time. At the same time there will not exist passage or flow of time with in it. Once the change of film happens, time flows within the film as well as film to film.

Thus in a space time (Minkowski) all the points will have different quantum states and each quantum state is without flow of time. So in that coordinate systems there exist points with constant time but vary with space. All these points describes the position of the point (contains information) in this universe. It describes an object at that point. But due to the fact that it is quantum point, it will not contain space. The combination of all these points provides positions of all points as a single film of the universe. Here the word "Combination" means "superposition". Thus "super position" of all these quantum states termed as a universal film describing a point in quantum space time or quantum coordinate system.

But we can not observe this quantum point since there will not be flow of time until unless goes from one film to another. So it is imaginary only.

This is the point where we have gone much profoundly than the quantum state interpreted mathematically as state vectors [18] and the wave function involved in Schrodinger wave equation [19]. In these mathematical descriptions, flow of time has been included. It is a hidden secret of nature. But when we consider the physical meaning of quantum states at the most fundamental level, a quantum state is a universal film in which time exists but flow of time will not be there. It is a standstill picture of space (further it is explained as information and time in quantum coordinates). Flow of time exists only when a film changes in to another film (in theory it is described by a mechanism of consciousness). 
Even Schrödinger wave equation (time dependent or independent) is also inclusive of flow of time. Obviously, since it is a wave, it must be a part of passage of time. But when it is divided further in to films where time will not exist (only space exist), the quantum state described by mathematics has no meaning. Means, even if it is a pure state, it is a mixture of two more states (which are termed as films). There is a hidden theory in between these purest states that involves origin of time and consciousness. This paper tried to provide that insight as a new model of consciousness

\section{Observation and the Reality}

Figure 3 represents the transformation to make time to flow. When time flows, imaginary quantum states will be super positioned and form a reality within the film and at the same time film also will be changed. Since it is working with a mechanism to form an internal superposition, film has to be changed. Thus it continues and shows any point in this space time coordinates with a specific special distance (non zero). Thus all the points of object observed as a reality of that object. Figure 3 illustrates that the super positioned quantum states at point $q_{s}$ in quantum coordinate system will be transformed as a real point $p_{r}$ in conventional relativistic frame.

Thus interpretation of observation or measurement of quantum states which

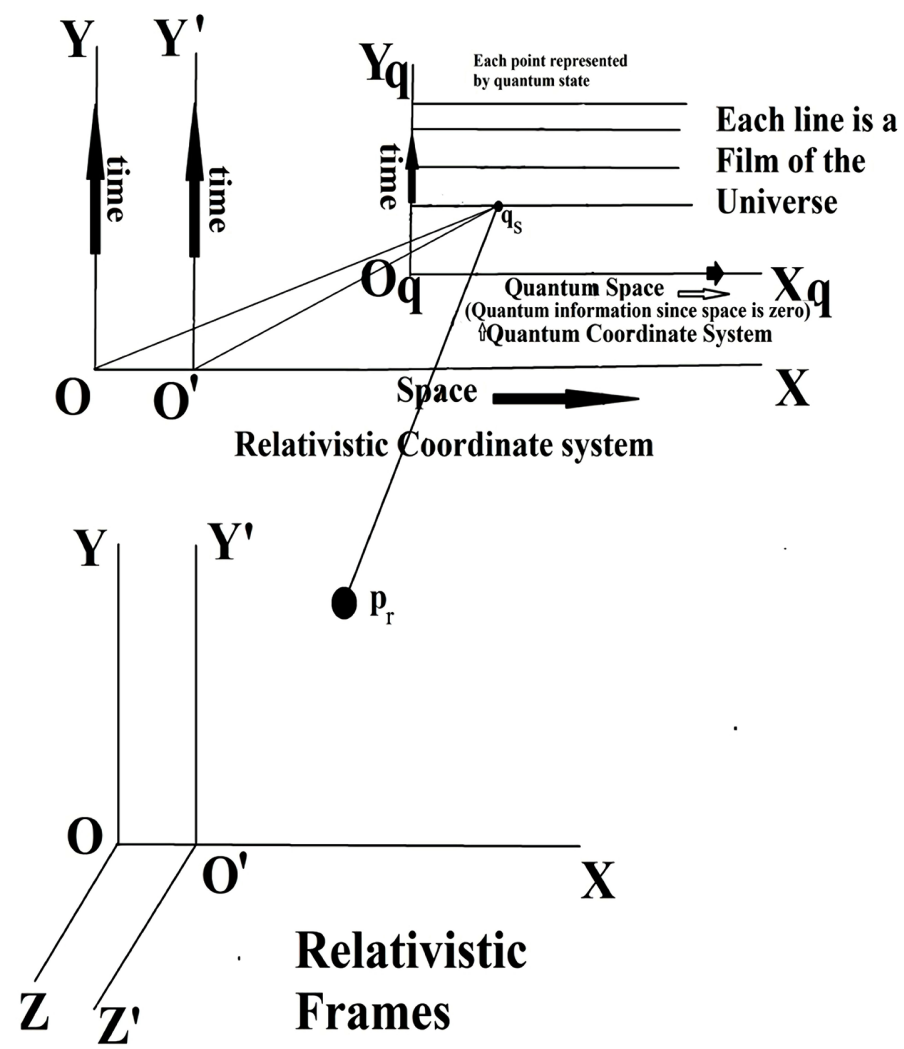

Figure 3. Transformation of quantum coordinate system to relativistic coordinate system in the process of observation. The point $q_{s}$ in quantum coordinate system will be transformed as the point $p_{r}$ in conventional relativistic frame as reality. 
are not real (imaginary only). The reality exists in transformation through the additional coordinate system called as "quantum coordinate system".

\section{Perspective of a New Model of Consciousness}

The physics of consciousness has been explained in "new hypothesis on consciousness" [11] elaborately. Actually it is not a hypothesis. It is an analysis of few fundamental queries of philosophy of physics like "why light velocity is constant for all inertial frames of reference?", "how space time originated?", "what is physical entity that makes difference between a living thing and a non living thing?" etc. It has concluded a preliminary model of consciousness integrating all the aspects explained in above sections of this article. It was similar to OrchOR Theory. But it has refuted the idea of quantum states described by mathematical support. Especially it is contradicting Orch-OR in emphasizing the fact that physical interpretation of quantum state and superposition of states is not relevant at the most fundamental level where time plays an important role. Now in this paper, it was substantiated by introducing quantum coordinate system that was hidden in the conventional transformations. The model has not been questioned much on biological or neurological aspects of the Orch-OR theory. But this paper says that no models or experiments on consciousness can sustain without considering these basics at quantum level.

Let us review the mechanism behind this new model of consciousness (Figure 4).

\subsection{Mechanism behind This Model}

Quantum mechanics interprets a point as space zero (time only will exist) [7].

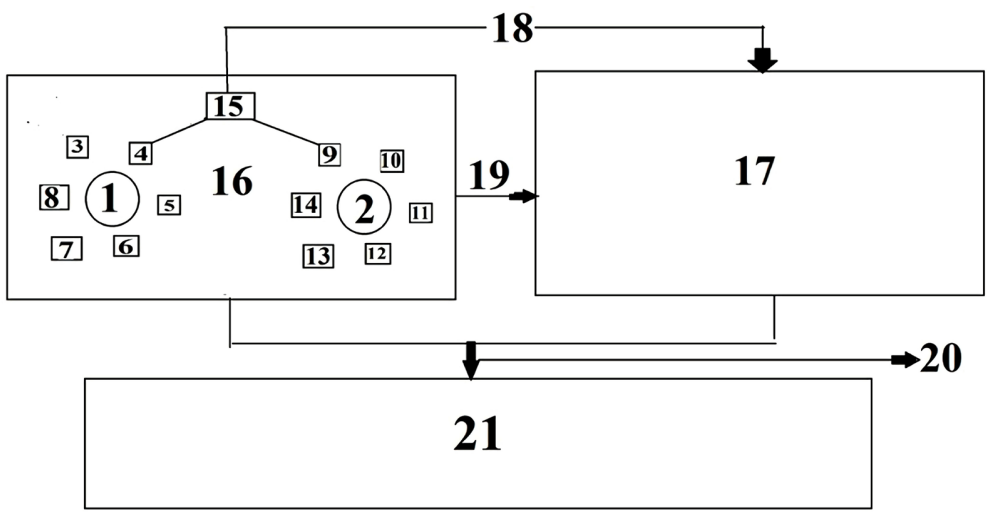

Figure 4.1 \& 2 are separate conscious observers; 3 to 8 are Quantum states associated to observer 1; 9 to 14 are quantum states associated to observer 2; 15 is Super positioned state of one state from observer with one state of observer 2 for example super positioned state of $4 \& 9 ; 16$ is Film containing observers $1 \& 2 ; 17$ is the change in film 16 as a part of this mechanism; 18 is the Process of mechanism that is to be completed for film change from 16 to 17 (It is the process of super position of $10^{42}$ quantum states of one observer with one quantum state of another observer); 19 is the Process of mechanism of film change from 16 to 17 . It is a consequential and simultaneous process of $18 ; 20$ is the Process indicating Superposition of films $16 \& 17 ; 21$ is the Final result, the reality. 
A specific time will exist for a specific point. If we interpret this in a quantum coordinate system, specific time will exist for all points on a line parallel to axis denoting space. Thus all the points can be shown with different space on the same line. Line parallel to space coordinate will have different space for each point on that line. But different space does not have a meaning because, according to quantum coordinates there will not exist distance or space. So even though there exist space, it is considered as zero space only. So instead of space coordinates, we can call it as information coordinates. On any parallel line, time will be constant but information will change (in other terms, space changes and the time also changes accordingly). So in order to keep time constant, the excess time will change in to space as per space time equivalence principle. Since space is zero, it will be denoted by change in information). "Zero space" between points means that all these points with different information are super positioned at a specific point. Thus that point is a superposition of all information nothing but superposition of all quantum states.

After overall view of this mechanism, the statement or the postulate that time is information [11] has to be modified as "the quantum of space time is information" according to space time equivalence principle, space and time can be convertible to each other. So to keep time as constant the space has to be converted in to information (information means, it may contain mass like quantities or observables associated to that quantum state). But when we transform it to relativistic coordinates, there will not be zero space point. Whatever small the point is, it contains some diameter. So the super positioned quantum states will be divided to a space and the point will be observed with some diameter. In other words any object can be observed with some length coordinate.

For example, in quantum coordinates, photon is a space zero super positioned quantum state. But in relativistic transformation, it will have space. Since relativity considered it as maximum velocity, as per its basic principle, its space must be zero. Since it is relativistic, it is the space between two divided quantum states. Conscious observer observes it again as super positioned quantum states. This is the final observation called as reality than the observation defined by physics without involvement of consciousness. This is the reality.

In quantum coordinate system "time" and "information" exists and in relativistic system both space and time exist. Means, time converts in to space. Here we have seen that in space time conversion, mass or charge also will be originated. Space time conversion phenomena elaborate it [16].

\subsection{Application of Double Relativity Effect to Consciousness}

There exists only one signal which is the maximum velocity and whose velocity is constant for all inertial frames in each space time. All fundamental forces will have their own space times and space time diameter can also be calculated [7]. This signal is a point in quantum coordinates with space zero but contains time and information. It will be mathematically represented as quantum state. Since it 
is a signal, its velocity is an absolute velocity (velocity is constant for all inertial frames of reference. For example, photon with velocity " $c$ " for our four dimensional universe). Observed velocity is the velocity which can be represented by Lorentz coordinates. As per "double relativity effect", the relation between observed velocity $v_{o}$ and absolute velocity $v_{a}$ is $v_{o}=v_{a} \gamma$. So the observed velocity " $c$ " will have an absolute velocity as $\frac{c}{\sqrt{2}}$ and $\gamma=\sqrt{2}$ since " $c$ " is the observed velocity follows the equation $v_{a} \gamma=c$. But without involvement of conscious mechanism as per Figure 2 and Figure 4, one cannot find the reality of the observed velocity. Thus, this observed velocity can be changed as reality as in Figure 5 .

Figure 5 explains three instances of observation.

\subsubsection{Instance-1}

Figure 5(a) shows the phenomena of observation in relativistic coordinates due to "double relativity effect". In that $v_{a}$ is absolute velocity and $v_{o}$ is observed velocity and are related by equation

$$
v_{o}=v_{a} \gamma
$$

where

$$
\gamma=\frac{1}{\sqrt{1-\left(\frac{v_{a}}{c}\right)^{2}}}
$$

Figure 5(a) illustrates the "double relativity equation" " $v_{o}=v_{a} \gamma$ ". And the relativistic factor " $\gamma$ ” for conventional relativistic (Galilean or Lorentz coordinates) coordinate system.

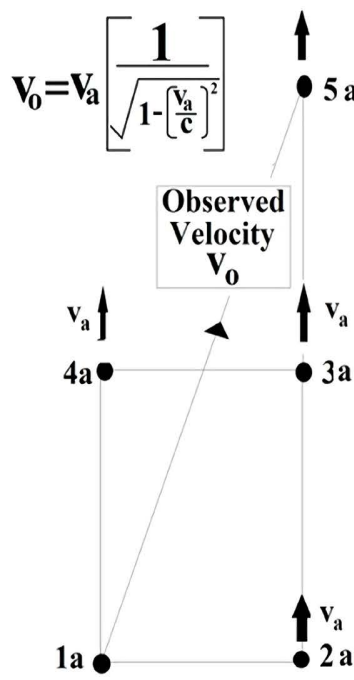

(a)
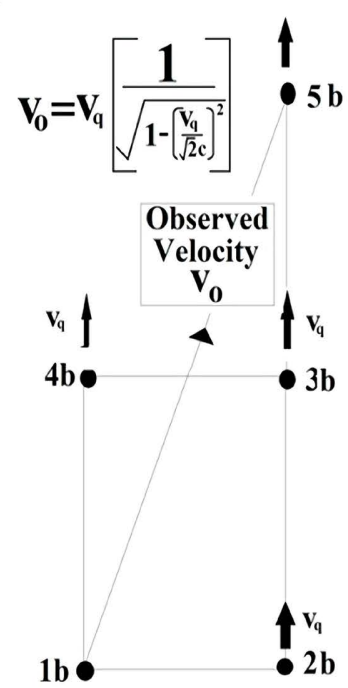

(b)

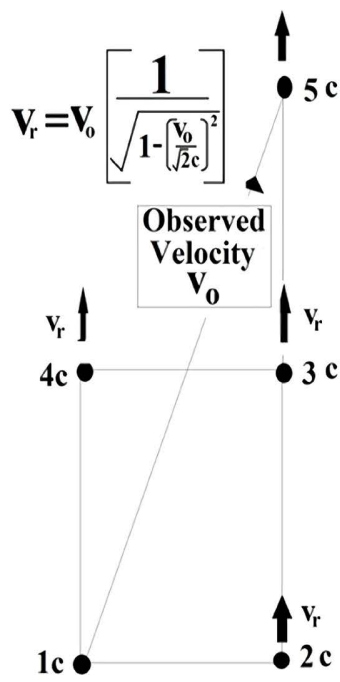

(c)

Figure 5. The reality is the consequence of double relativity effect and consciousness on the process of observation. (a) Observation in relativistic coordinates; (b) Observation in Quantum coordinates; (c) Final reality in the process of observation. 


\subsubsection{Instance-2}

The same observation in quantum coordinates has been shown in Figure 5(b). As explained above (Section 2.1) quantum coordinates contains points in space time coordinates with time coordinate only and the space coordinate is always zero. Its quantum particle is "bio force" particle [7] [15] and signal velocity is $\sqrt{2} c$. Thus the same special relativity principle is applied and all the velocities are comparable to that velocity. Here absolute velocity and observed velocity are also related by $v_{o}=v_{a} \gamma$ but here $\gamma=\frac{1}{\sqrt{1-\left(\frac{v_{a}}{c \sqrt{2}}\right)^{2}}}$ it represents consciousness frame of super relativity [15]. Figure 5(b) shows the quantum coordinates. So the absolute velocity $v_{a}$ can be replaced with $v_{q}$. The same has been explained in Figure 6 now we can rewrite the quantum relativistic factor $\gamma_{q}=\frac{1}{\sqrt{1-\left(\frac{v_{q}}{c \sqrt{2}}\right)^{2}}}$

$$
\therefore v_{o}=v_{q} \gamma_{q}
$$

where

$$
\gamma_{q}=\frac{1}{\sqrt{1-\left(\frac{v_{q}}{c \sqrt{2}}\right)^{2}}}
$$

Figure 5(b) illustrates the "double relativity equation" $v_{o}=v_{q} \gamma_{q}$ and relativistic factor $\gamma_{q}$ for quantum coordinate system.

These two instances (instances $1 \& 2$ ) are not relevant when we use consciousness mechanism's affect on observation. So the observed velocity in Figure 5 (c) is a combination of these two instances and the special relativity should not be violated. So the same "double relativity effect" explains it as $v_{o}$ is observed velocity and it is the reality, where $\gamma_{r}=\frac{1}{\sqrt{1-\left(\frac{v_{o}}{c \sqrt{2}}\right)^{2}}}$.

$$
\therefore v_{r}=v_{o} \gamma_{r}
$$

where

$$
\gamma_{r}=\frac{1}{\sqrt{1-\left(\frac{v_{o}}{c \sqrt{2}}\right)^{2}}}
$$

Thus $\gamma_{r}$ satisfy both the instances.

Figure 5(c) illustrates the "double relativity equation" " $v_{o}=v_{q} \gamma_{q}$ " and relativistic factor " $\gamma_{q}$ " as absolute reality due to the affect of consciousness on observation.

This has been elaborated in Figure 6. It shows a quantum point with respect to coordinates and its position inside that point. The reality is combination of both. 


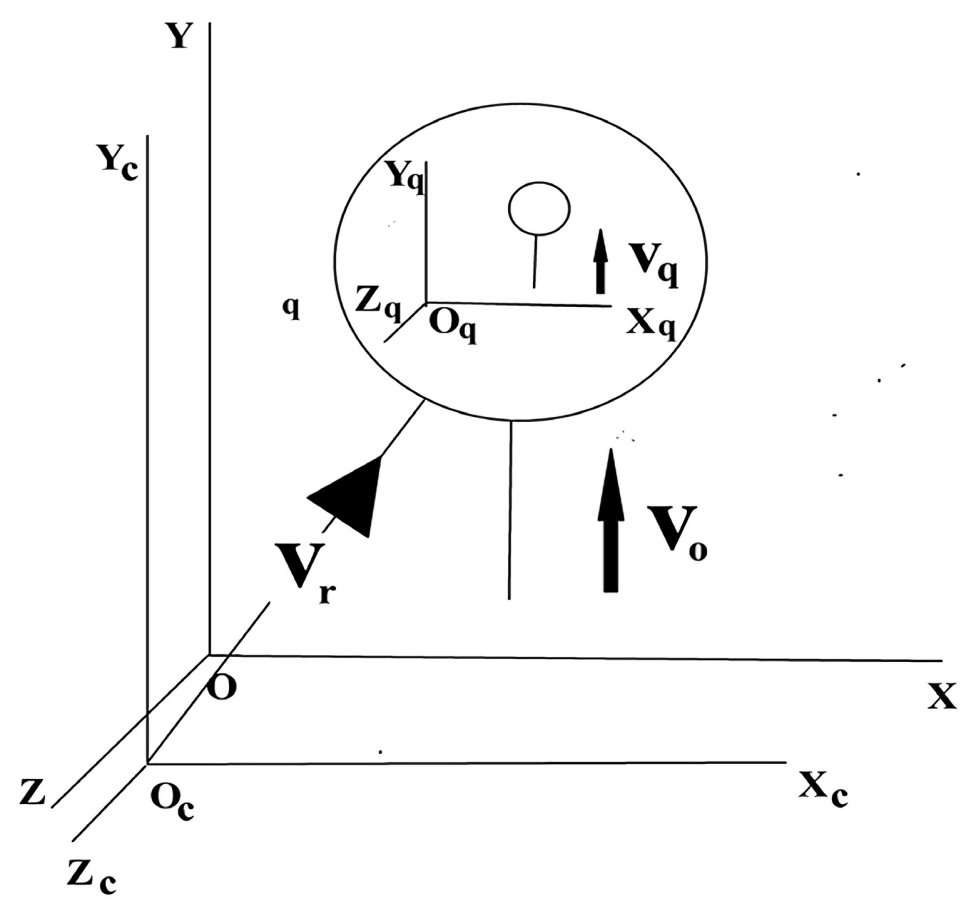

Figure 6. Role of consciousness in relativistic interpretation of quantum point.

With respect to XYZ frame the circle is a point and the space inside is zero. This coordinate system is conventional Lorentz coordinate system obeying special theory of relativity. The velocity shown is $v_{o}$. The signal velocity is " $c$ " as it follows special relativity. The same point with respect to the coordinate system $X_{q} Y_{q} Z_{q}$ inside circle i.e. quantum coordinate system, for which space coordinate is zero for all points. Only time coordinate exist. And the velocity is $v_{q}$ and follows the equation $v_{o}=v_{q} \gamma_{q}$ where $\gamma_{q}$ is according to equation (4). Conscious observer will be in different frame $X_{c} Y_{c} Z_{c}$. With reference to conscious observer at origin $O_{c}$, the velocity is $v_{r}$ and follows Equations (5) \& (6).

Finally, we can say frame of observation will be affected due to "consciousness" and the frame will be considered as different from observers frame of reference defined in conventional relativistic (Galilean or Lorentz coordinates). Due to "double relativity effect" and "affect of consciousness on observation" the signal velocity expected to become $c \sqrt{2}$ and the relativistic factor will be changed accordingly.

Calculation of this $\sqrt{2}$ is also an important aspect of consciousness which was elaborated in [15]. Now we can emphasize it in simple way.

Simply the logic behind this is.

If the relativity factor for "double relativity effect" and "special relativity" is same, the equation can be written as

$$
\gamma=\frac{1}{\sqrt{1-\left(\frac{v_{a}}{c}\right)^{2}}}
$$


In double relativity effect $v_{o}=v_{a} \gamma$ so " $c$ " will be $c \gamma$ since " $c$ " is also absolute velocity. So equation can be written as $\gamma=\frac{1}{\sqrt{1-\left(\frac{v_{o}}{c}\right)^{2}}}$. Here signal velocity is $c \gamma$ and observed velocity remains $v_{o}$. Therefore we can rewrite the equation as $\gamma=\frac{1}{\sqrt{1-\left(\frac{v_{o}}{c \gamma}\right)^{2}}}$.

If we solve it, $\gamma$ will be $\sqrt{2}$. Now the signal velocity is $c \sqrt{2}$ and the Equations (5) \& (6) are applicable.

It is illustrated by Figure 5(c) as the reality of observation. Thus the final reality is an overall affect of "double relativity effect" on "consciousness" and observation. Figure 5 illustrated it. It is not possible to observe these velocities without involvement of consciousness. So the result is a proof for consciousness also.

Finally, in the process of observation, fist the quantum coordinates have to be changed to Lorentz. So double relativity effect must be used. Then these points are compatible with Lorentz coordinates. It is called observation and velocity is observed velocity. This will undergo the process of consciousness mechanism and become reality. So again relativity must be applied with respect to conscious observer. The signal velocity is $c \sqrt{2}$. All velocities must be compared to it. And velocity is observed velocity and follows Equation (5). So this is the final reality for which relativistic factor $\gamma_{r}$ follows the Equation (6). This factor is different from Lorentz relativistic factor. Here velocity $v_{o} \leq c \sqrt{2}$. Velocity $v_{r}$ is the real velocity which is $\gamma_{r}$ times than the observed velocity " $v_{o}$ ". Observed velocity is limited to Lorenz coordinates. Real velocity is due to the effect of consciousness on observed velocity. So the consequences like relativistic kinetic energy etc are also different. This result will supersede relevant conclusions of the paper [17] and proposes a profound understanding of the problem. This can be observed by any particle physics laboratory. Specifically these affects can be observed on the observed velocities between $c \sqrt{2}$ to $c$.

It is not possible to observe these velocities without involvement of consciousness. So the result is a proof for consciousness also.

\section{New Perspective on Synchronization of General Relativity with Quantum Mechanics}

\subsection{Involvement of Concept of Time}

Einstein-Hilbert action is a similar approach as a least action principle in which least action is treated as a point in space time and the equation shows a graviton if it converges. Till now it has considered from the point of general relativity. So there are suspicious views of scientists on this approach [20]. Perturbation in basically fixed point is fine at larger distances but quantum mechanically the gravitational interaction is irrelevant [9]. Not only that, the point they considered as least action is not space zero mass zero. In this Cartesian system it must 
have some space. Zero space cannot be considered in this system whereas space zero can be considered in quantum system. But when it converts in to space in this coordinate system, it will have space time diameter with a least diameter that converges due to Hilbert action.

\subsection{Relevance of Time Operator in Quantum Mechanics}

It seems relevant to mention an idea [21] regarding interpretation of "time operator" while discussing these issues. The mathematical methodology the author adopted is good and helpful to develop the conceptual interpretations described in this paper. Otherwise, the author can utilize the theoretical background to interpret the most basic level of quantum state with respect to the involvement of "time operator" proposed by him. The present paper demands the necessity of a Schrödinger equation without a "time operator" or with a zero value. Thus the present paper may lead to an exact and most relevant time independent Schrödinger wave equation to reveal some of the secrets of this nature.

\subsection{Relevant to Other Interpretations}

Other interpretations like Random discontinuous motion (RDM) interpretation of Quantum Mechanics [22] [23] or "particle interference" rather than wave interference to describe wave function collapse [24] etc. are also must be originated from the conceptual conclusions of the present paper. It elaborates the evolution of time and its involvement in superposition of two time independent quantum states to form space time which is the root cause for the formation of particle or wave.

\section{Comparison with Other Models of Consciousness}

This model is different from other theories of consciousness in

1) Observation is not for object only. Object is a part of the universal film as a whole at a particular time.

2) Minimum two observers are required to generate one conscious movement of any observer.

\subsection{Observer Effect and Necessity of Conscious Observer}

On double slit, observer's effect changes the reality. It raised a question on the necessity of conscious observer. Most of the scientists ruled out the point that observer must be conscious [4] [25]. But, as per this paper, observer means conscious observer only. Detector is also part of conscious observer. Instead of detector if we provide conscious observer, that is also not sufficient to accept the affect of conscious observer on observation. The model of consciousness presented in this paper and its mechanism emphasizes on the fact that there must be two conscious observers to observe the physical entities existed in this universe. The transformations of the interactions between quantum states involved in this observation and super position of these states considered as reality which 
is more than a simple observation. The change in these interactions with time only will provide wave function and its collapse which turns a particle in to wave and vice versa. So this experiment explains only the measurement problem. It is not connected to consciousness and observation. Two conscious observers must exist to get conscious experience of the effect of observation or reality.

\subsection{Refute of the Idea That Consciousness Is Everywhere in This Universe}

Few authors are denying the idea that consciousness is attributable to living things only. They are saying that consciousness belongs to whole universe [4] [25] [26] of course there is an argument against it [27] which says that wave function collapsed somewhere and all in one. My paper is supporting that idea and contradicts that consciousness is everywhere. And clearly says that consciousness is for living things only. Because, detector is also part of observation and the conscious observer only can find detector. This can easily understand by double slit experiment. If we provide a detector in between slit and the beam generator, the wave function will be collapsed. If we switch of it, it will not be collapsed. Means, the universal films of observer is a super position of quantum states of the objects signal generator, double slit screen and the detector in the process of signal generation to the screen via slit. It will be collapsed and behaves like a particle if detector is on. If it is switched off, the screen shows interference pattern because the detector finds the reality in to one "eigen state" just like conscious observer. If it is switched off, it is not super positioned as explained for conscious observer. Here there is no observation by conscious observer so when detector switches off, interference pattern occurs. If conscious observer is there in the place of detector, the same results will come. That means, the mechanism is same for a detector as well as conscious observer (Detector itself will not exist without conscious observers). Detector itself is a superposition of lot of quantum states or universal films. So if it is divided in to single states, there will not be existence at all. Again conscious observer is required to create the superposition of quantum states of all the objects and the process.

\subsection{This Consciousness Model \& the Quantum Eraser Delayed Choice Experiment}

One of the outcomes of delayed choice quantum eraser physically means that "the future will change past" Big question is how is it possible?

It is possible only when the observer is conscious and it is completely interlinked with total universe instead of a particular object as explained in the paper [11] where universal film is made up of a loop connecting all the conscious observers only. Non living things or objects are created and experienced by these conscious observers connected in this loop. The loops can be connected from one to another depending upon their time frame. The model elaborated in this paper explains that all the detectors are objects only and they are part of the conscious process generated by conscious observer. So here in this experiment 
also all the detectors can be viewed as conscious observers.

Here in this experiment, a photon splits in to a pair of entangled photons. One is delayed than the other. As per this model of consciousness, in the observation of delayed pair of photon, the quantum states super positioned at a point in quantum coordinates and the entangled pair with another point. Quantum coordinates denote a point with time and information. So, for these two pairs the time and information is different. But to observe each pair each observer is required and observer's time is different. If time is different, it cannot be in same film. These two observers must be in the same film if the entangled pair is of one particular photon. Then the process will be continued to next film. So as per this model there is no meaning in "past" and "future" in within these two. Only the "present" exists with combined effect. Whatever change comes to one pair will change another. If we come out of the box of this experiment, whole universe is existed like that. Film of total universe is connected to the loop of conscious observer [11] and the loop will change its connection as per its inertial frame of reference, whenever requires and goes to past films or to future films. It is nothing but time travel. This is the explanation for the question "whether the future will change past?"

\subsection{Explanation to Grandfather Paradox by This Model}

Grandfather paradox is also an important aspect of "physics of time travel" and "freewill" concepts of philosophy. Few authors [28] elaborated the closed loops of time travel within the scope of existing theories.

According to this model of consciousness,

1) Conscious observer is necessary for the existence of reality of the universe. Reality of the materialistic universe is due to interaction of quantum states of two conscious observers. There may exist lot of films in between these interactions.

2) Two conscious observes create one more conscious observer. There exist several connected films in a sequential order which obey cause and effect law of physics. So any of these two conscious observes erased by any reason before creation of the new conscious observer, the interlinked sequential order of the films will be erased and new conscious observer will be erased. There exist lot of other quantum states of this erased conscious observer but they are all imaginary only since reality requires a devise like brain or a centralized network connecting all. There are lot of films in between interaction of two observers to create a conscious observer with such devise. Without such devise, the interactions are not realistic. So to form this erased observer in to reality there must exist interaction which creates this devise otherwise the connective reality will be erased with the new observer.

3) If it is applied to grandfather paradox. If a grandson of a grandfather goes to past and kills his grandfather, he will also be vanished since the connective films after the death of grandfather will be erased from reality. Grandson is also 
part of that reality. Now both the devises grandfather and grandson are compatible to each other. So grandson vanished. Compatible means both are in reality. Above model says that only one reality exists. If one more reality with one more devise exist, both cannot be compatible. All other are imaginary only. Thus the time travel will work out within the compatible devises or one reality only. Thus, if devises are not vanished because of each other, time travel is possible by regulating their devise. Only present will exist for that devise and it is the only reality. For others it may be viewed as past and present. Grandson can change all materialistic things in future and present but it cannot change the interaction of conscious observers who are reason for his birth.

4) With reference to new hypothesis [11] consciousness is a circuit or a loop connecting to conscious observers only [Refer fig. 7 Page no.39]. Here in this paper it is substantiated as reality. So one observer may connect to another observer in so many ways. New observers in this circuit can also be created due to interaction of two observers. Thus a new circuit can also be created like branches from original and sub branches to main branches. In this if any of the main branch erases, all the sub branches will also be erased. Thus in grandfather paradox, grandfather is the main branch and sub branches are father and mother. Grand son is sub branch to father and mother. Here if he kill his grandfather, whatever may be other connections of the grandson, all the connected branches to his grandfather will be erased. And the grandson and his all other connections will be erased.

5) But in some theories it is told that grandson is a copy of the original went to past and other alternate of the universe will be started after killing of grandfather. But here it is only one reality and connects only to conscious observers. without conscious observers the universe will not exist.

6) Thus films with zero time and infinite time are not possible.In between so many films will exist. Finally two conscious states are must to create reality. Thus, those two conscious observers are eternal whatever may be their time frame is. Other conscious observers are created from them. Materialistic creation is due to the interaction of these conscious observers only.

7) Devise and regulators are important for consciousness.

8) Two conscious observers created this space time. At that stage they might have a separate device than us. Later they created several conscious observers with different devises.

9) Now we have a devise called brain and it will have a regulator. If we go back to past, when we have not born, the devise will not be there. So everything connected to this devise will be lost. Devise connection is reality, means, through devise only we can see reality.

10) So we cannot go to future where we will not exist (our consciousness will not exist) and cannot go to past where we have not created (born).

11) Devise will have reality. Reality will have past and future. Another devise is required to see beyond us. So for one devise, the universe will not exist but 
universe will exist for other devises.

12) Totally universe exists always as it is with quantum information. Its superposition is time.

13) We will see the universe through devise in a streamlined way otherwise that is only haphazardly distributed information and time only. Since there will not be space it is a simple point from where the time emerges.

14) So if we kill our grandfather in past we will not exist. We will be vanished and universe also will not exist simply it is nothing not even a space.

\section{Conclusions}

1) The word "observation" of physics has been redefined and it is emphasized on the fact that observation is attributed to Lorentz coordinates only. Till now physics says that "observation" and "measurement" in these conventional coordinates are taken granted as reality. But actual reality is different from this observation. Quantum mechanics is not reality. It remains imaginary until unless it converts into relativistic coordinates. Simply applying relativity to quantum mechanics as in the case of "relativistic quantum mechanics" is not relevant. The physical meaning of quantum states is connected to the problem of time and formation of space time. So the involvement of consciousness is unavoidable in observation or measurement. Thus the imaginary quantum states first have to be interpreted in terms of conventional coordinate system. Prior to transforming these quantum states into conventional coordinate system, "double relativity effect" is to be applied. Due to the application of double relativity effect the observation changes and the reality will be different from observation. The ultimate result says that factor Lorentz relativistic factor " $\gamma$ " has to be modified as $\gamma_{r}=\frac{1}{\sqrt{1-\left(\frac{v_{o}}{c \sqrt{2}}\right)^{2}}}$. So the observables will be modified than the results of special theory of relativity and the results will be different within the range of velocities $c \sqrt{2}$ to " $c$ ". This is can be verified in any laboratory.

2) Application of this concept may provide new path to researchers working on velocities more than that of light and the affect of drastic change in momentum on Einstein's field equations towards singularity. The synchronization of quantum mechanics and relativity applied in this paper indicates that General Relativity equations may be applicable to lower diameters comparable to Planck length up to zero space.

3) A new model of consciousness has been proposed by emphasizing the fact that conscious observer plays an important role in observation. It also proposed that there must exist minimum two conscious observers to find the reality. It explains the concept of origin of time and formation of space time and its curvature at the basic level (in other terms its quantum level). Thus it explains the synchronization between quantum mechanics and relativity.

4) Totally it concludes a major change in observation and reality which can be 
observed in any lab. Thus if it is proved experimentally, it will be the best proof for this model of consciousness.

\section{Conflicts of Interest}

The author declares no conflicts of interest regarding the publication of this paper.

\section{References}

[1] Casado, C.M.M. (2008) Latin-American Journal of Physics Education, 2, 152. http://www.journal.lapen.org.mx,p-152

[2] Deutsch, D. (1985) International Journal of Theoretical Physics, 24, 1-41. https://link.springer.com/article/10.1007/BF00670071 https://doi.org/10.1007/BF00670071

[3] Fields, C. (2012) Information, 3, 92-123. https://doi.org/10.3390/info3010092

[4] Yu, S. and Nikoli, D. (2010) Quantum Mechanics Needs No Consciousness (and the Other Way Around). https://arxiv.org/pdf/1009.2404.pdf

[5] Schlosshauer, M., Koer, J. and Zeilinger, A. (2013) A Snapshot of Foundational Attitudes toward Quantum Mechanics. https://arxiv.org/pdf/1301.1069.pdf

[6] Sinha, K.P., Sivaram, C. and Sudarshan, E.C.G. (1976) Foundations of Physics, 6, 6570. https://link.springer.com/article/10.1007/BF00708664 https://doi.org/10.1007/BF00708664

[7] Kodukula, S.P. (2019) Journal of Modern Physics, 10, 466-476. https://doi.org/10.4236/jmp.2019.104032

[8] Carlip, S. (2001) Quantum Gravity: A Progress Report. https://arxiv.org/pdf/gr-qc/0108040.pdf

[9] Shomer, A. (2007) A Pedagogical Explanation for the Non-Renormalizability of Gravity. https://arxiv.org/pdf/0709.3555.pdf

[10] Hameroff, S. and Penrose, R. (2014) Physics of Life Reviews, 11, 39-78, 41, 48, 49, 59. https://doi.org/10.1016/j.plrev.2013.08.002

[11] Kodukula, S.P. (2019) International Journal of Physics, 7, 31-43. http://pubs.sciepub.com/ijp/7/2/1

[12] Mihelic, F.M. (2019) Experimental Evidence Supportive of the Quantum DNA Model. Proceedings SPIE, Quantum Information Science, Sensing, and Computation XI, Vol. 10984, 1098404. https://doi.org/10.1117/12.2517348

[13] Kodukula, S.P. (2009) Double Relativity Effect \& Film Theory of the Universe. Lulu.com, Raleigh, 5-6, 7-12, 13-32.

[14] Kodukula, S.P. (2009) Heart of the God with Grand Proof Equation-A Classical Approach to Quantum Theory. Lulu.com, Raleigh.

[15] Kodukula, S.P. (2014) American Journal of Modern Physics, 3, 232-239. http://article.sciencepublishinggroup.com/pdf/10.11648.j.ajmp.20140306.15.pdf https://doi.org/10.11648/j.ajmp.20140306.15

[16] Kodukula, S.P. (2021) Journal of High Energy Physics, Gravitation and Cosmology, 7, 1333-1352. https://doi.org/10.4236/jhepgc.2021.74083

[17] Kodukula, S.P. (2017) International Journal of Physics, 5, 99-109. http://pubs.sciepub.com/ijp/5/4/1 
[18] Weinberg, S. (2012) Collapse of the State Vector. https://arxiv.org/pdf/1109.6462.pdf

[19] Schrodinger, E. (1926) The Physical Review, 28, 1049-1070. https://journals.aps.org/pr/pdf/10.1103/PhysRev.28.1049 https://doi.org/10.1103/PhysRev.28.1049

[20] Padmanabhan, T. (2008) International Journal of Modern Physics D, 17, 367-398. https://doi.org/10.1142/S0218271808012085

[21] Routh, A.K. (2019) Open Access Library Journal, 6, e5816. https://doi.org/10.4236/oalib.1105816

[22] Gao, S. (2020) Foundations of Physics, 50, 1541-1553. https://doi.org/10.1007/s10701-020-00390-0

[23] Wechsler, S.D. (2021) Journal of Quantum Information Science, 11, 99-111. https://doi.org/10.4236/jqis.2021.113008

[24] Niehaus, A. (2019) Journal of Modern Physics, 10, 423-431. https://doi.org/10.4236/jmp.2019.104027

[25] De Barros, J.A. and Oas, G. (2017) Foundations of Physics, 47, 1294-1308. https://doi.org/10.1007/s10701-017-0110-7

[26] Tononi, G. and Koch, C. (2015) Philosophical Transactions of the Royal Society B, 370, Article ID: 20140167. https://doi.org/10.1098/rstb.2014.0167

[27] Reason, C.M. (2017) Comment on the Paper Quantum Mechanics Needs No Consciousness by $\mathrm{Yu}$ and Nikolic (2011). https://arxiv.org/abs/1707.01346

[28] Lobo, F. and Crawford, P. (2003) Time, Closed Time like Curves and Causality. https://arxiv.org/pdf/gr-qc/0206078.pdf 